\title{
GuÍA BÁSICA \\ Para el programa de envejecimiento de aeronaves de la Fuerza Aérea Colombiana
}

En esta investigación se realiza el priLmer acercamiento que se hace de manera formal para tratar el problema del envejecimiento de aeronaves. Por lo tanto, ¿Es necesario diseñar una guía de doctrina básica para estandarizar los programas de envejecimiento de aeronaves que se desarrollen en la FAC?

El soporte teórico de este planteamiento se centró en los antecedentes de envejecimiento de la aeronaves en el mundo, los reportes de los programas de envejecimiento, los accidentes causados por el envejecimiento de los sistemas no estructurales como el cableado que ha generado una serie de accidentes como el Boeing 747-100 y el caso ALOHA.

Asímismo, se destacan que los elementos que hacen parte del sistema estructural de las aeronaves se ven afectados por los factores de estrés, las cargas aerodinámicas, la corrosión que se exponen por el contacto continuo de los factores ambientales, el diseño de materiales y los tiempos de operación destinados para cada componente.

En este contexto se aplicó la técnica de revisión documental en la Jefatura de Operaciones Logística, Dirección de Mantenimiento y Grupos Técnicos para identificar las actividades y tareas de los operadores de las aeronaves en cuanto al seguimiento de los problemas relacionados con el envejecimiento y se recopiló información especializada que permitiera identificar la situación en la Fuerza Aérea.

El análisis de la anterior información demostró que a pesar que el envejecimiento es una temática actual que ha obligado a desarrollar cambios significativos desde hace veinte años para la institución, es un tema que sólo hasta hace seis años se empezó a trabajar.

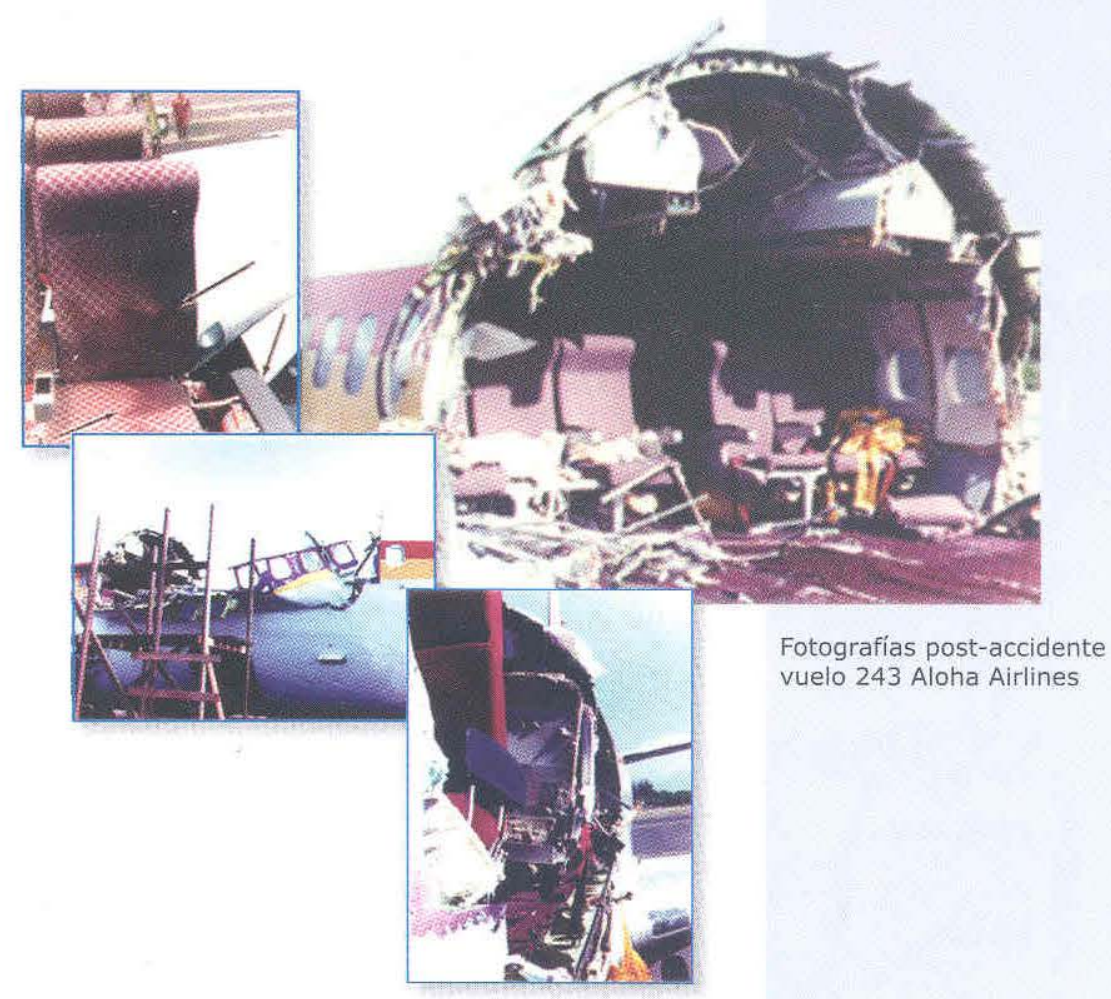

Los programas de mantenimiento ordenados por la casa fabricante no garantizan la operación segura de las aeronaves; por lo tanto, se requiere que el operador complemente estos programas mediante nuevas tareas de mantenimiento generadas por el monitoreo y análisis del comportamiento de los sistemas de la aeronave. Esto obliga a que la Fuerza Aérea investigue y desarrolle tareas teniendo en cuenta el diseño y configuración.

A pesar de que las misiones y estrategias acerca de las operaciones aéreas cambian, no se ha realizado de manera paralela una modernización de todas las flotas de las aeronaves, por lo que es importante tener poca variedad de equipos y más cantidad de aeronaves que puedan cumplir con las diferentes misiones típicas que facilitaran el soporte logístico y la continuidad de los programas de envejecimiento de las flotas de las aerona- 


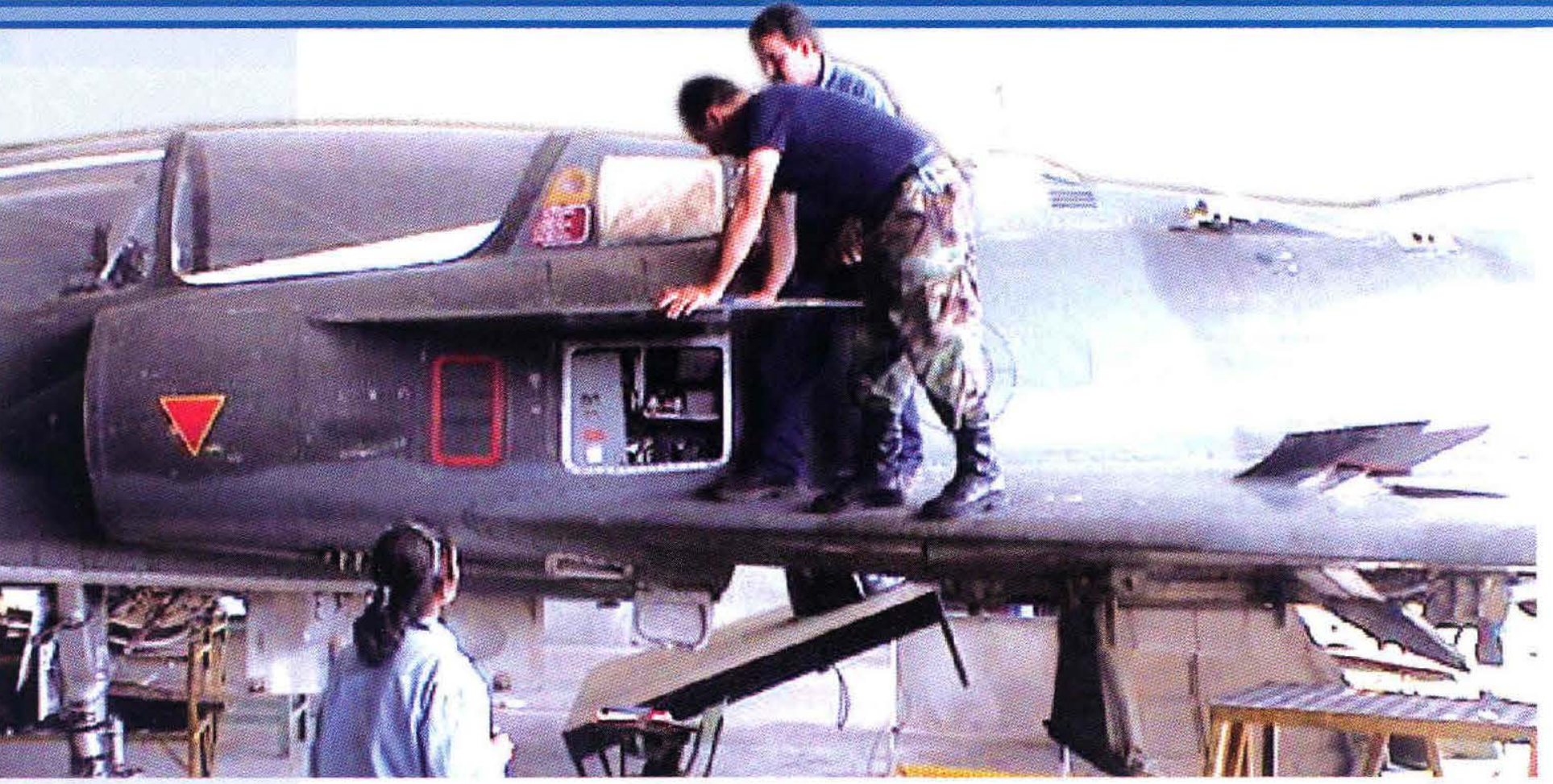

ves. Con base en estos resultados se diseñó la guía básica para el programa de envejecimiento de aeronaves que contiene IDENTIFICACIÓN GENERAL DEL PROYECTO

\begin{tabular}{|c|c|c|}
\hline PROGRAMA & $\begin{array}{l}\text { ESPECIALIZACIÓN EN LOGÍSTICA } \\
\text { AERONÁUTICA }\end{array}$ & $\begin{array}{l}\text { gias y he- } \\
\text { rramientas }\end{array}$ \\
\hline TÍTULO & $\begin{array}{l}\text { GUÍA BÁSICA PARA EL PROGRAMA DE } \\
\text { ENVEJECIMIENTO DE AERONAVES DE } \\
\text { LA FUERZA AÉREA COLOMBIANA }\end{array}$ & $\begin{array}{l}\text { para gene- } \\
\text { rar la base }\end{array}$ \\
\hline AUTORES & $\begin{array}{l}\text { CT. CÉSAR ROMERO PRADILLA } \\
\text { MY.JUAN DAVID BLANCO CAUSIL } \\
\text { MY. PEDRO ARNULFO SÁNCHEZ SUÁREZ }\end{array}$ & $\begin{array}{l}\text { doctrinaria } \\
\text { de mante- } \\
\text { nimiento }\end{array}$ \\
\hline $\begin{array}{l}\text { LÍNEA DE } \\
\text { INVESTIGACIÓN }\end{array}$ & DOCTRINA & $\begin{array}{l}\text { que permi } \\
\text { tirá inicia }\end{array}$ \\
\hline $\begin{array}{l}\text { ÁREA } \\
\text { FUNCIONAL }\end{array}$ & EDUCACIÓN AERONÁUTICA & $\begin{array}{l}\text { programa } \\
\text { de enveje }\end{array}$ \\
\hline GESTOR & JEFE DE PLANEACIÓN-JOA & $\begin{array}{l}\text { cimiento } \\
\text { todos }\end{array}$ \\
\hline
\end{tabular}

el contenido se encuentran los siguientes temas: Seguridad operacional en el envejecimiento de las aeronaves con el criterio de confiabilidad. Estrategias que van desde configurar sistemas de información confiable hasta establecer los indicadores. Consideraciones para el programa de envejecimiento que contienen los factores que afectan el envejecimiento de los sistemas de las aeronaves como el estrés y factores de carga y corrosión. Las acciones del programa de envejecimiento con cumplimiento de los estándares y procedimientos técnicos, verificación del comportamiento, registros de datos, acciones correctivas y sistemas de información. $Y$ por último la autoevaluación de los procesos del control de envejecimiento con la herramienta de diagnóstico para la gestión.

Se espera que la anterior guía se incluya en el Manual de Mantenimiento FAC, para que sea aplicada en los grupos técnicos, con opción de ser modificada para una mejor implementación y desarrollo de tareas en prevención del envejecimiento de las aeronaves.

Finalmente, se recomienda que la Fuerza Aérea Colombiana aproveche los convenios existentes entre Estados Unidos y Colombia para realizar la transferencia en tecnología y conocimientos que ha desarrollado la FAA y la USAF, mediante los programas de intercambio de los oficiales de mantenimiento. 


\section{Manual Operacional de Armamento Aéreo}

El manual se enmarca en el ámbito de los niveles superiores en esta materia.

El estudio se encuentra contextualizado en la doctrina táctica con base en documentos similares emitidos por la Fuerza Aérea Colombiana, como por ejemplo el Manual Técnico de Armamento Aéreo, y otras fuentes mundiales que son autoridades en el tema.

En estos documentos se encuentran las diferentes directivas, boletines y órdenes permanentes emitidas por el Comando de la Fuerza Aérea, dónde se establece de qué for- rencia obligado para el desarrollo de futuras aplicaciones clasificadas en el nivel más específico de la doctrina? Para dar respuesta a la anterior pregunta se hace necesario replantear y documentar la doctrina táctica acerca de la utilización del armamento para ser más efectivos en el combate $y$ en el momento de operarlos. De ahí, que se realizó un estudio descriptivo que permitió la recopilación de la información para efectuar las descripciones de las características y tipos de armamento utilizados, el rendimiento y los diferentes programas para la toma de decisiones.

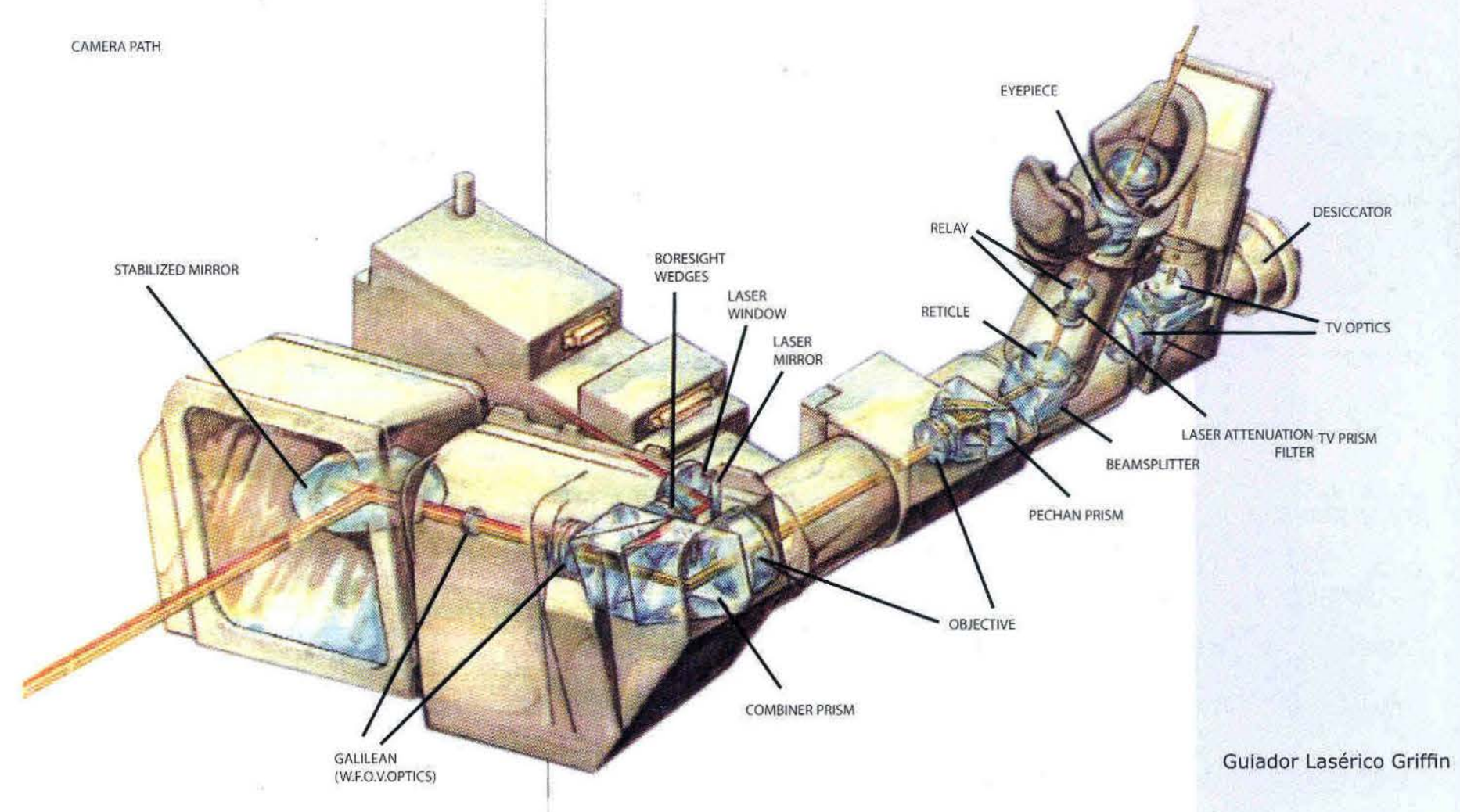

ma y en qué momento se utilizarán los medios aéreos y su respectivo armamento en las diferentes misiones. Entonces, ¿cuál debería ser el complemento de la doctrina básica y táctica, que se convierta en un punto de refe-
Así el contenido del manual presenta una historia del armamento en la Fuerza Aérea, un capítulo con las definiciones más utilizadas en el campo del armamento, la organización para el combate que tiene como ob- 


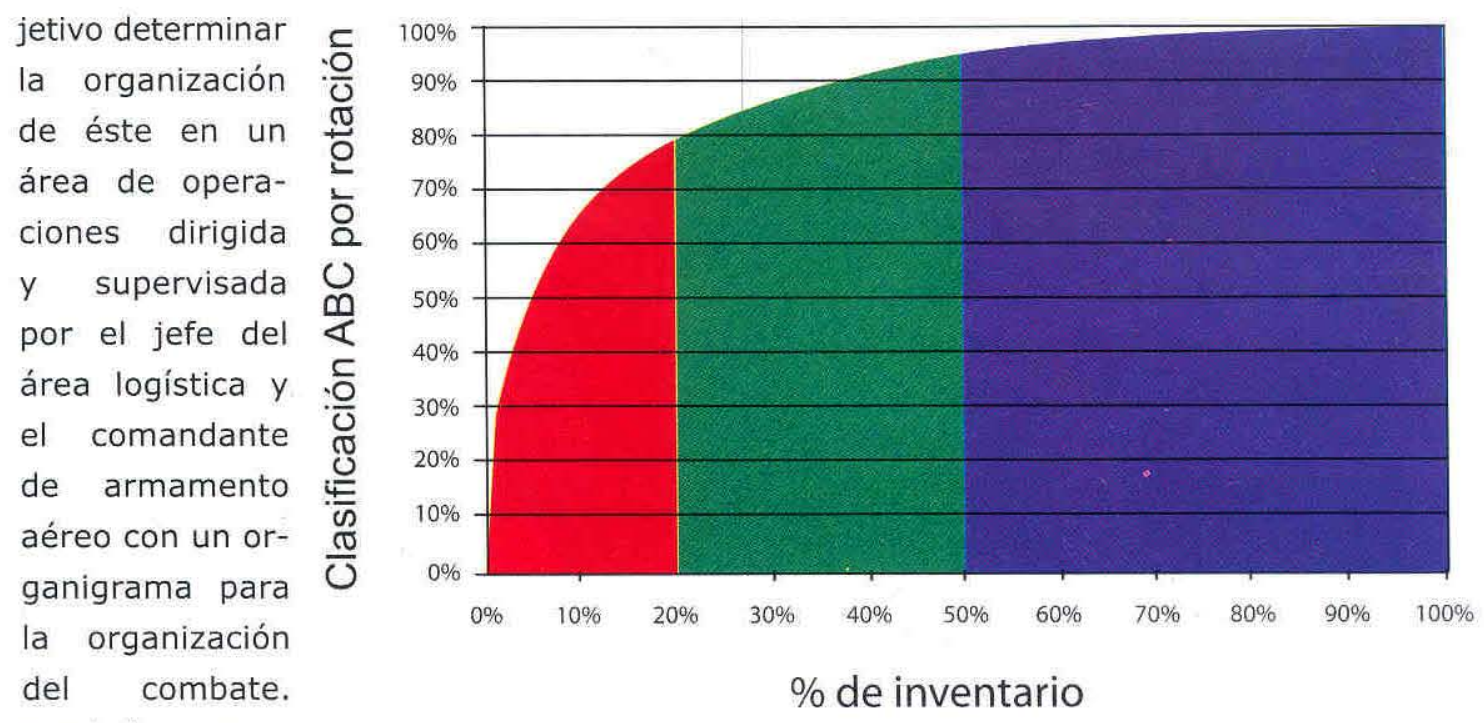

También se pre-

En el aparte de destrucción de objetivos sentan las normas de seguridad y entrenamiento para el personal de armamento. Entre ellas se encuentran normas de seguridad y manipulación del material de guerra y el entrenamiento del personal de arma-

\section{IDENTIFICACIÓN GENERAL DEL PROYECTO}

PROGRAMA

ESPECIALIZACIÓN EN LOGÍSTICA AERONÁUTICA

TÍTULO

MANUAL OPERACIONAL DE ARMAMENTO AÉREO

AUTORES Capitán JOSÉ LUIS CONDE BARBERY Capitán JUAN CARLOS RUEDA

CARTAGENA

Capitán GUILLERMO ZUÑIGA SANTANA

LÍNEA DE

INVESTIGACIÓN ARMAMENTOAÉREO

ÁREA

FUNCIONAL LOGÍSTICA AERONÁUTICA

GESTOR JEFEDE PLANEACIÓN-JOL

AÑO

2003 mento aéreo.

En la segun-

da parte se encuentra el capitulo uno que establece el sistema de armas entre los que está el sistema de armamento compuesto por el sistema de entrega de armas. La información se complementa con gráficos, figuras y dibujos de los diferentes tipos de armas. Igualmente, se describen los daños de las municiones y explosivos con los efectos destructivos según la clasificación de las municiones utilizadas según el objetivo. se presentan las generalidades y su correspondiente clasificación como los objetivos en el mar con tablas y cuadros; los objetivos en tierra igualmente con datos, tablas y gráficas.

La tercera parte del manual se refiere a los helicópteros donde presenta los siguientes modelos: H-500, AB212, AH60L. De acuerdo con este parámetro describe las características, las normas que deben conocer las tripulaciones y las reglas de seguridad. En el capitulo de aviones los clasifica según los siguientes modelos: M-5, K-FIR, A-37, AT-27, AC-47, TOV-10, que se utilizan para el comandante con el planeamiento de una operación de tal forma que hace la referencia de las características, las capacidades, configuraciones, los sistemas y tipos de armas. Asímismo, presenta la lista de chequeo del comandante para el planeamiento de una operación, el uso del armamento aéreo y las configuraciones típicas de los escuadrones de aeronaves según el modelo.

Con el compendio de este manual se establece una doctrina táctica, se estandariza la utilización de las aeronaves de acuerdo con el tipo de armamento y se tiene una panorámica completa con sus respectivos impactos. 